\title{
BMJ Open Cardiovascular mortality and oral antidiabetic drugs: protocol for a systematic review and network meta- analysis
}

Rashmi Goyat, ${ }^{1}$ James Douglas Thornton, ${ }^{2}$ Xi Tan, ${ }^{1}$ George A Kelley ${ }^{3}$

To cite: Goyat $R$, Thornton JD, Tan $\mathrm{X}$, et al. Cardiovascular mortality and oral antidiabetic drugs: protocol for a systematic review and network meta-analysis. BMJ Open 2017;7:e017644. doi:10.1136/ bmjopen-2017-017644

- Prepublication history and additional material for this paper are available online. To view these files, please visit the journal online (http://dx.doi. org/10.1136/bmjopen-2017017644).

Received 5 May 2017

Revised 16 October 2017

Accepted 31 October 2017

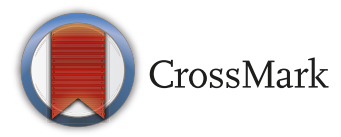

${ }^{1}$ Department of Pharmaceutical Systems and Policy, School of Pharmacy, West Virginia University, Morgantown, West Virginia, USA

${ }^{2}$ Department of Pharmaceutical Health Outcomes and Policy, College of Pharmacy, University of Houston, Houston, Texas, USA ${ }^{3}$ Department of Biostatistics, School of Public Health, West Virginia University, Morgantown, West Virginia, USA

Correspondence to

Rashmi Goyat;

ragoyat@mix.wvu.edu

\section{ABSTRACT}

Introduction Cardiovascular diseases are the leading cause of morbidity and mortality among individuals with diabetes. Despite the beneficial effects of antidiabetic drugs (ADDs) in terms of lowering haemoglobin A1c, several ADDs have been shown to increase the risk of cardiovascular events. Given the high prevalence of cardiovascular disease among individuals with diabetes, it is important to weigh the benefits of ADDs against their cardiovascular safety. Therefore, the objective of the current study is to conduct a systematic review with network meta-analysis to compare the effects of different oral pharmacological classes of ADDs on cardiovascular safety.

Methods and analysis Randomised clinical trials (RCTs) and observational studies published in English up to 31 January 2017, and which include direct and/or indirect evidence, will be included. Studies will be retrieved by searching four electronic databases and cross-referencing. Dual selection and abstraction of data will occur. The primary outcome will be cardiovascular mortality. Secondary outcomes will include all-cause mortality, new event of acute myocardial infarction, stroke (haemorrhagic and ischaemic), hospitalisation for acute coronary syndrome and urgent revascularisation procedures. Risk of bias will be assessed using the Cochrane Risk of Bias assessment instrument for RCTs and the Strengthening the Reporting of Observational Studies in Epidemiology instrument for observational studies. Network meta-analysis will be performed using multivariate random-effects meta-regression models. The surface under the cumulative ranking curve will be used to provide a hierarchy of ADDs that increase cardiovascular mortality.

Dissemination The results of this study will be presented at a professional conference and submitted to a peerreviewed journal.

PROSPERO registration number CRD42017051220.

\section{INTRODUCTION}

\section{Rationale}

Diabetes is a global public health problem, with prevalence expected to increase from 382 million to 592 million people by $2035 .{ }^{1}$

\section{Strengths and limitations of this study}

To the best of our knowledge, this is the first systematic review with network meta-analysis that compares the cardiovascular safety of different classes of antidiabetic drugs based on data from both randomised clinical trials (RCTs) and observational studies.

- Common to most meta-analyses, significant and unexplained heterogeneity may exist.

- Like any aggregate data meta-analysis, the risk for ecological fallacy exists.

- Few RCTs may report data on cardiovascular mortality.

It is the seventh leading cause of death in the USA, with cardiovascular (CV) disease being the most common cause of death from diabetes. ${ }^{2}$ Importantly, type 2 diabetes mellitus (T2DM) more than doubles the risk of CV events. ${ }^{34}$ Annual per-person medical care cost of treating adults with T2DM and CV disease is US $\$ 10$ 172, approximately two times greater than the cost of treating T2DM alone. ${ }^{5}$

Pharmacotherapy is the mainstream strategy to manage diabetes by controlling haemoglobin A1c (HbA1c), which is important to reduce the risk of $\mathrm{CV}$ events and premature mortality. ${ }^{67}$ However, in 2007, the safety of rosiglitazone, a member of the thiazolidinedione class of antidiabetic drugs (ADDs), was questioned. ${ }^{8}$ Specifically, rosiglitazone was found to be associated with an increased risk of heart attacks, stroke and mortality from $\mathrm{CV}$ events. ${ }^{8}$ In addition to rosiglitazone, the $\mathrm{CV}$ safety of several other ADDs, including pioglitazone and sulfonylureas, has been questioned. ${ }^{9-15}$ For example, while dipeptidyl peptidase-4 inhibitors (DPP-4Is) were shown to be associated with decreased hospitalisation due to heart failure in one study, ${ }^{16}$ another study reported a 
higher rate of hospitalisation due to heart failure among those using DPP-4Is. ${ }^{17}$ Based on the potential deleterious consequences of ADDs and subsequent CV events, the US Food and Drug Administration (FDA) revised their policy in 2008 to place greater emphasis on examining CV safety for all trials testing newer ADDs. ${ }^{18}$ However, the effects of ADDs on CV events, including CV mortality, have not been firmly established. ${ }^{8}{ }^{10}$ To the best of the authors' knowledge, only one previous network meta-analysis has been conducted with respect to the CV safety of ADDs. ${ }^{19}$ In this study, authors included randomised clinical trials (RCTs) that were 24 weeks or longer. No statistically significant difference was reported between CV mortality and any drug class. While these findings are encouraging, the inclusion of only RCTs may be potentially limiting, given that prior to 2008, ADDs were only required to prove clinical efficacy in terms of lowering HbAlc. As a result, the clinical trials of the drugs that received regulatory approval before 2008 may not have reported CV outcomes. Consequently, a need exists for a meta-analysis that includes both RCTs and observational studies so that adverse outcomes can be appropriately documented. In addition, it has recently been suggested that RCTs and observational studies should not be considered in isolation. ${ }^{20}$ Furthermore, additional studies may have been published since the previous studies search for eligible trials (21 March 2016). ${ }^{19}$ Given the former, a need exists for an updated network meta-analysis that also includes observational studies.

\section{Objective}

The primary objective of this study is to conduct a systematic review with network meta-analysis of randomised trials and observational studies to compare the effects of different pharmacological classes of ADDs on CV mortality. The network meta-analytic approach is appropriate here because it allows for the inclusion of multiple interventions from both direct and indirect comparisons that have not been examined in a head-to-head fashion. ${ }^{21}$

\section{METHODS}

\section{Overview}

This study will follow the Preferred Reporting Items for Systematic Reviews and Meta-Analyses guidelines ${ }^{22}$ for meta-analyses of healthcare interventions and the current protocol report follows the Preferred Reporting Items for Systematic Reviews and Meta-Analyses Protocols. ${ }^{23}$ This protocol is registered in International Prospective Register of Systematic Reviews (trial registration number: CRD42017051220).

\section{Eligibility criteria}

Studies that meet the following criteria will be included: (1) randomised trials and observational studies; (2) adults $\geq 18$ years of age with T2DM, either with or without a history of CV disease; (3) at least one oral
ADD intervention group; (4) comparative control group for randomised trials that do not include more than one oral ADD; (5) data on CV mortality and/ or major adverse cardiac events; (6) studies published in English up to 31 January 2017. The decision to include patients with T2DM with or without a history of CV disease was made based on our preliminary search of clinical trials that included patients with either a history of $\mathrm{CV}$ disease or those who are at a heightened risk for CV disease. Because T2DM and a history of CV disease are both associated with an increased risk of acute myocardial infarction (AMI) and stroke, ${ }^{24}$ we decided to include patients with T2DM with or without a history of CV disease and compare new events of AMI and stroke between the two groups. Such criteria will also allow for selected comparisons. A decision was made to only include oral ADDs because there are two classes of injectable antidiabetic medications approved for treatment in the USA. These include insulin and glucagon-like peptide-1 receptor agonists. Typically, insulin and other injectable drugs are not first-line therapies in the treatment of T2DM. These are prescribed to more severe patients who are not able to achieve appropriate glucose levels with other therapies. Therefore, to keep the study population more uniform, only oral antidiabetic agents will be included.

Direct comparisons will be made from the studies comparing two or more ADDs and indirect comparisons will be made from studies comparing an ADD to a placebo. Both randomised clinical trials and observational studies will be included because randomised clinical trials were not required to include CV outcomes before 2008, and it has recently been suggested that RCTs and observational studies should not be looked at in isolation. ${ }^{20}$ Major adverse cardiac events will be defined as an incidence of AMI, stroke, hospitalisation for acute coronary syndrome and urgent revascularisation procedures. These measures were identified by the US FDA to ascertain the CV safety of ADDs in the regulatory guidelines issued for the industry. ${ }^{18}$

\section{Information sources}

The following databases will be searched from their inception forward for potentially eligible studies in English language published on or before 31 January 2017: (1) PubMed, (2) Scopus, (3) Web of Science, (4) Cochrane Central Register of Controlled Clinical Trials, (5) clinical trials registry (ClinicalTrials.gov). In addition, cross-referencing from retrieved studies will be conducted.

\section{Search strategy}

Search strategies adapted from a previous research ${ }^{19}$ will be developed using text words and Medical Subject Headings. Electronic databases will be searched for studies on the effects of oral ADDs on CV safety in adults with T2DM. The first author will conduct all database searches. A preliminary search strategy for PubMed is shown in online supplementary file 1 . The search strategy for all 
other databases will be adapted based on the requirements of each database.

\section{Study records}

Study selection

All studies extracted from electronic databases using the search strategy will be imported into EndNote V.X7.5. Duplicate studies will be removed electronically using the 'Find Duplicates' tool in EndNote. The studies will be examined again manually to find and delete any additional duplicates. The first two authors will select studies independent of each other. Complete articles will be obtained for all titles and abstracts that appear to meet the inclusion criteria or where there is any uncertainty. Reasons for exclusion will be coded as one or more of the following: (1) inappropriate population, (2) inappropriate intervention, (3) inappropriate comparison, (4) inappropriate outcome(s), (5) inappropriate study design and (6) other. After selection, the first two authors will review their selections and resolve any discrepancies by consensus. If consensus cannot be reached, the third author will be consulted. The overall agreement rate prior to correcting discrepant items will be calculated using Cohen's kappa ( $\kappa)$ statistics. Once discrepancies are resolved, the overall precision of searches will be calculated by dividing the number of studies included by the total number of studies screened after removing duplicates. The number needed to read will then be calculated as the inverse of the precision. A flow diagram that depicts the search process and an online supplementary file that includes a reference list of all studies excluded (including the reason(s) for exclusion) will be included in the study. The proposed structure for the flow diagram is shown in figure 1 .

\section{Data abstraction}

Before initiating data abstraction, a codebook will be developed in Microsoft Excel 2013. The codebook will be developed by the first author with input from the third author. The major categories of variables to be coded will include: (1) study characteristics (author, journal, year, etc); (2) participant characteristics (age, sex, HbAlc, CV disease at baseline, etc); (3) intervention characteristics (pharmacological class of ADDs, dose, route of administration, etc); (4) control characteristics; (5) outcome data for CV mortality, all-cause mortality, incidence of AMI, stroke, hospitalisation for acute coronary syndrome and urgent revascularisation procedures. The first two authors will abstract data from selected studies, independent of each other, using the codebook in Microsoft Excel. On completion, both authors will review the codebooks and resolve discrepancies by consensus. If consensus cannot be reached, the third author will provide a recommendation. Prior to correcting disagreements, the overall agreement rate will be calculated using Cohen's $\kappa$ statistic.

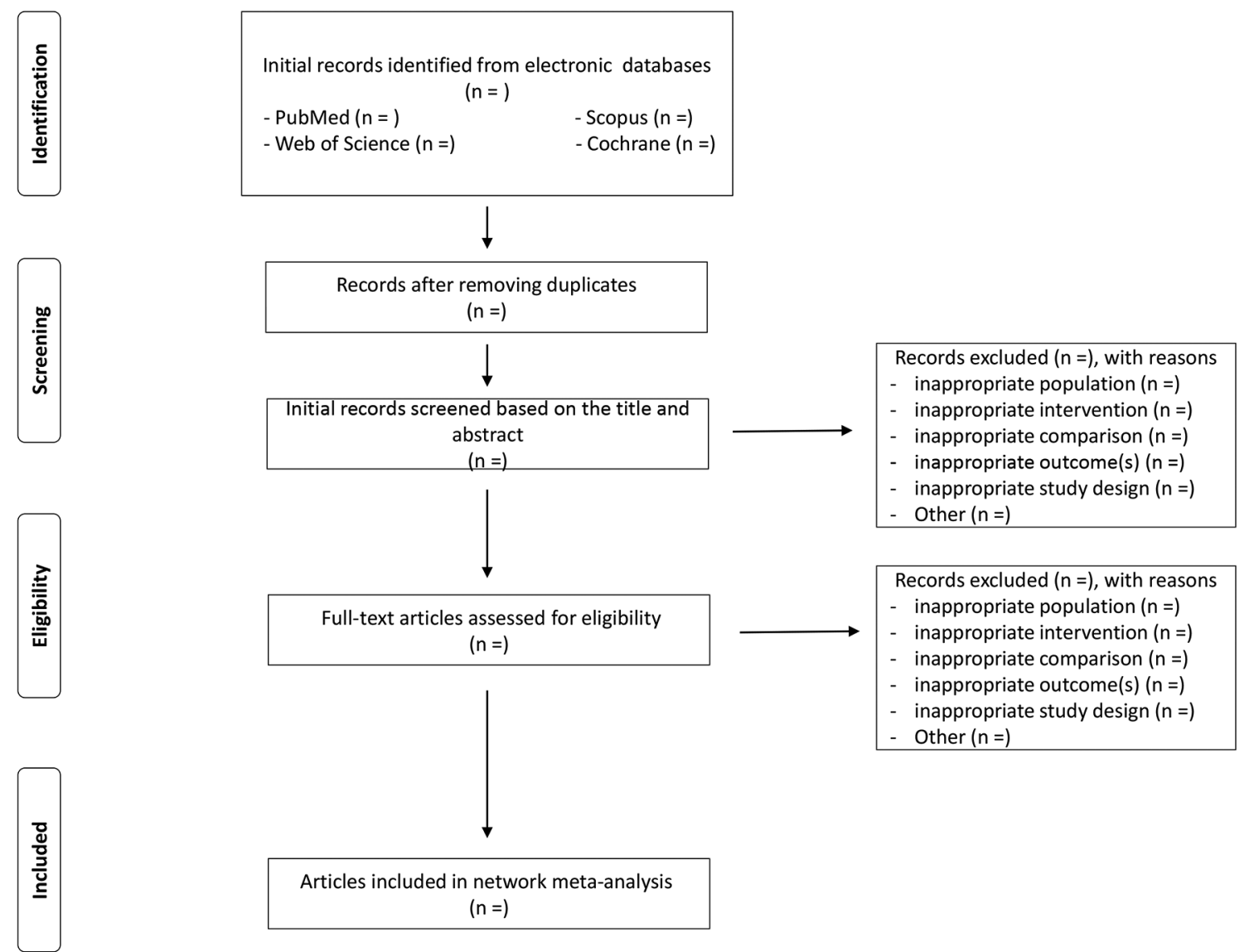

Figure 1 Flow diagram of study selection process. 


\section{Outcomes and prioritisation}

The primary study outcome will be CV mortality. Secondary outcomes will include all-cause mortality, new event of AMI, stroke, hospitalisation for acute coronary syndrome and urgent revascularisation procedures.

\section{Risk of bias assessment in individual studies}

Risk of bias for RCTs will be assessed using the Cochrane Risk of Bias instrument. ${ }^{25}$ Observational studies will be assessed using the Strengthening the Reporting of Observational Studies in Epidemiology (STROBE) instrument. ${ }^{26}$ Bias in RCTs will be evaluated for six domains: (1) random sequence generation, (2) allocation concealment, (3) blinding of participants and personnel, (4) blinding of outcome assessors, (5) incomplete outcome data and (6) selective reporting. Each study will be classified as having a high, low or unclear risk of bias overall and for each domain. The overall risk of bias will be classified as high if any one of the domains is considered high risk. The STROBE instrument is a checklist of 22 items that evaluates the quality of reporting for observational studies. No study will be excluded based on the results of risk of bias assessment. ${ }^{26}$ The first two authors will conduct all risk of bias assessments independent of each other. The two authors will then review the results for risk of bias assessment and resolve any discrepancies by consensus. If consensus cannot be reached, the third author will be consulted.

\section{Data synthesis}

\section{Calculation of effect sizes}

All analyses will be conducted using the natural log of OR and then transformed back to ORs for presentation purposes. If OR is not reported, it will be calculated from data reported in the study. If data are not available to calculate OR, it will be requested from the study authors. Secondary outcomes will be calculated using the same procedure as for our primary outcome. If a study includes both direct and indirect comparisons, only direct comparison data will be included given that the primary focus of the current study is to compare the CV safety between different ADDs. The data augmentation approach will be used to make direct comparisons if the control group is placebo. ${ }^{27}$ In this technique, direct evidence studies that lack a control (placebo) group will have one generated from the weighted average of the arm-specific means and SD. ${ }^{28}$

\section{Pooled estimates for change in outcomes}

Network maps will be drawn to depict the treatments that are directly compared against each other and the amount of evidence available for each treatment and its comparator. Separate network maps will be presented for each outcome. Contribution plots for each outcome will be generated to determine the most dominant comparisons for each network estimate, as well as for the entire network. The weights applied will be a function of the variance of the direct treatment effect and the network structure, the product being a per cent contribution of each direct comparison to each network estimate. Network and contribution plots will be produced using the networkplot and netweight commands, respectively, ${ }^{29}$ in Stata/IC for Mac V.14.0 (STATA; 2016).

Prior to conducting network meta-analysis, pairwise meta-analysis using random-effects models will be conducted in order to examine statistical heterogeneity within each comparison. ${ }^{21}$ Heterogeneity will be assessed using Cochran's Q statistics and $\mathrm{I}^{2}$, an extension of $\mathrm{Q} \cdot{ }^{30} 31$ A Q statistic $\leq 0.10$ and/or an $\mathrm{I}^{2}$ value $>50 \%$ will be considered to represent significant heterogeneity. On completion of pairwise meta-analysis, network meta-analysis will be performed using multivariate random-effects models based on the mumeta command in Stata/IC for Mac V.14.0. ${ }^{32}$ Non-overlapping $95 \%$ CIs will be considered to represent statistically significant changes. Separate network meta-analysis models will be used to compare CV mortality, all-cause mortality, incidence of AMI, stroke, hospitalisation for acute coronary syndrome and urgent revascularisation procedures.

Sub-group analyses will be conducted to examine the association between our primary outcome and oral ADDs. These will include year of drug approval by the US FDA, presence or absence of $\mathrm{CV}$ disease risk at baseline, HbA1c at the baseline, number of comorbidities, type of treatment (monotherapy, dual therapy or triple therapy) and the country the study was conducted in. Secondary outcomes will be handled using the same approach.

We will examine the consistency of the estimates of treatment effects from direct and indirect evidence for each outcome using the mvmeta command in Stata. ${ }^{28}$ An alpha value $\leq 0.05$ will be considered to represent statistically significant inconsistency. Prediction intervals will be used to enhance the interpretation of findings and provide an estimate of expected results in a future study. ${ }^{28}$ Prediction intervals will be generated using the mvmeta and interval plot ${ }^{29}$ commands in Stata/IC for Mac V.14.0.

\section{Meta-biases}

Small-study effects (publication bias, etc) will be assessed using comparison-adjusted funnel plots. Unlike traditional funnel plots in pairwise meta-analysis, funnel plots in network meta-analysis need to account for the fact that studies estimate treatment effects for different comparisons. Consequently, there is no single reference line from which symmetry can be evaluated. For the comparison-adjusted funnel plot, the horizontal axis will represent the difference between study-specific effect sizes from the comparison-specific summary effect. In the absence of small-study effects, the comparison-adjusted funnel plot should be symmetric around the zero line. Since the treatments need to be organised in some meaningful way to examine how small studies may differ from large ones, comparisons will be defined so that all refer to an active treatment versus a control group. Comparison-adjusted 
Table 1 Covariates that will be included in the study

\begin{tabular}{|c|c|}
\hline Characteristics & Variables \\
\hline Study & $\begin{array}{l}\text { Publication year, country the study was conducted in, type of study (RCT, cohort, case-control, etc), } \\
\text { duration of the study, follow-up duration. }\end{array}$ \\
\hline Intervention & Name of the drug, pharmacological class, dose, route of administration. \\
\hline Comparator & Name of the drug, pharmacological class, dose, route of administration. \\
\hline
\end{tabular}

$\mathrm{AMI}$, acute myocardial infarction; HbA1c, haemoglobin $\mathrm{A} 1 \mathrm{c} ; \mathrm{RCT}$, randomised clinical trial.

funnel plots will be generated using the netfunnel command $^{29}$ in Stata/IC for Mac V.14.0.

Transitivity (similarity in the distribution of potential effect modifiers across the different pairwise comparisons $)^{33}$ will be evaluated using random-effects network meta-regression while controlling for the different study designs within each comparison. Potential effect modifiers will include age, gender, baseline HbAlc, duration of T2DM, obesity, presence of CV disease at baseline and medication status. In addition, because individuals taking medication are more likely to have severe disease or more comorbidity than those without medication, we will also include baseline condition of the patient (eg, disease severity) in our regression models. However, since this is an aggregate data meta-analysis and if the patients included within each study are heterogeneous (eg, different levels of disease severity within the same study), we will include as a covariate those studies that control for such factors versus those that do not. Table 1 provides a complete list of covariates that we plan to include. Transitivity analysis will be conducted using the mometa command $^{28}$ in Stata/ IC for Mac V.14.0.

Ranking analysis is a major advantage of network meta-analysis because it allows one to rank all interventions for the outcome of interest. For the current study, we will generate ranking plots for a single outcome using probabilities. $^{34} 35$ However, since ranking treatments based solely on the probability of each treatment being the best does not account for the uncertainty in the relative treatment effects and the potential for assigning higher ranks in which little information is available, rankograms and cumulative ranking probability plots will be used to show ranking probabilities along with their uncertainty for changes in our primary and secondary outcomes. ${ }^{34} 35$ Surface under the cumulative ranking curves (SUCRA), a transformation of mean ranks, will be used to provide a hierarchy of treatments while accounting for the location and variance of all treatment effects. ${ }^{34}{ }^{35}$ Larger SUCRA values are indicative of better ranks for the treatment. Separate ranking analyses will be conducted for all primary and secondary outcomes using the mumeta ${ }^{28}$ and SUCRA ${ }^{29}$ commands in Stata/IC for Mac V.14.0.

\section{Software used for data synthesis}

All data will be analysed using Stata/IC for Mac V.14.0.

\section{Confidence in the cumulative evidence}

Strength in the body of evidence will be assessed using the Grading of Recommendations Assessment, Development and Evaluation (GRADE) instrument for network meta-analysis. ${ }^{36}$ Two main outputs are reported in a network meta-analysis: pairwise effect estimates and treatment rankings. Since the two outputs are generated using different techniques, they may differ between each other. Therefore, it is important to assess the level of confidence to be placed on each output. The level of confidence will be assessed using GRADE across four domains: (1) study limitations, (2) joint consideration of indirectness and transitivity, (3) joint consideration of statistical heterogeneity and statistical inconsistency, (4) imprecision and publication bias. Based on these assessments, the overall strength of evidence will be ranked as either high, moderate, low or very low. The overall confidence will be classified as high if any one of the domains is considered high.

\section{REGISTRATION}

In accordance with Primary Reporting Items for Systematics Reviews and Meta-Analyses Protocols (PRISMA-P,) our systematic review with network meta-analysis was registered with the International Prospective Register of Systematic Reviews (PROSPERO) on 15 April 15, 2017 (registration number: CRD42017051220).

Contributors RG is the guarantor. RG, JDT, XT and GAK drafted the manuscript. $\mathrm{RG}, \mathrm{JDT}, \mathrm{XT}$ and GAK contributed to the (1) development of the data sources to search for relevant literature, including search strategy, (2) selection criteria, (3) data extraction criteria and (4) risk of bias assessment strategy. GAK provided statistical expertise while RG, JDT and XT provided content expertise on mortality and antidiabetic drugs. All four authors read, provided feedback and approved the final manuscript.

Funding GAK was supported by the National Institute of General Medical Sciences of the National Institutes of Health (NIH), grant number U54GM104942. JDT is supported by the NIH Research Training Program in Behavioral and Biomedical Sciences, grant number T32GM081741-08.

Disclaimer The content of this manuscript is solely the responsibility of the authors and does not necessarily represent the official views of the National Institutes of Health. 
Competing interests None declared.

Provenance and peer review Not commissioned; externally peer reviewed.

Author note The protocol was amended on 6 April 2017 to update the list of team members. If this protocol is amended again, we will provide the date of each amendment, describe the change, and provide the rationale for the change.

Open Access This is an Open Access article distributed in accordance with the Creative Commons Attribution Non Commercial (CC BY-NC 4.0) license, which permits others to distribute, remix, adapt, build upon this work non-commercially, and license their derivative works on different terms, provided the original work is properly cited and the use is non-commercial. See: http://creativecommons.org/ licenses/by-nc/4.0/

(c) Article author(s) (or their employer(s) unless otherwise stated in the text of the article) 2017. All rights reserved. No commercial use is permitted unless otherwise expressly granted.

\section{REFERENCES}

1. Lorber D. Importance of cardiovascular disease risk management in patients with type 2 diabetes mellitus. Diabetes Metab Syndr Obes 2014;7:169-83.

2. American Diabetes Association. Statistics About Diabetes. 2016 http://www.diabetes.org/diabetes-basics/statistics/ (accessed 17 Feb 2017).

3. Schernthaner G. Cardiovascular mortality and morbidity in type-2 diabetes mellitus. Diabetes Res Clin Pract 1996;31:S3-13.

4. King P, Peacock I, Donnelly R. The UK prospective diabetes study (UKPDS): clinical and therapeutic implications for type 2 diabetes. $\mathrm{Br}$ $J$ Clin Pharmacol 1999;48:643-8.

5. Nichols GA, Brown JB. The impact of cardiovascular disease on medical care costs in subjects with and without type 2 diabetes. Diabetes Care 2002;25:482-6.

6. Xu L, Chan WM, Hui YF, et al. Association between HbA1c and cardiovascular disease mortality in older Hong Kong Chinese with diabetes. Diabet Med 2012;29:393-8.

7. Holman RR, Paul SK, Bethel MA, et al. 10-year follow-up of intensive glucose control in type 2 diabetes. N Engl J Med 2008;359:1577-89.

8. Hiatt WR, Kaul S, Smith RJ. The cardiovascular safety of diabetes drugs--insights from the rosiglitazone experience. $N$ Engl J Med 2013;369:1285-7.

9. Lago RM, Singh PP, Nesto RW. Congestive heart failure and cardiovascular death in patients with prediabetes and type 2 diabetes given thiazolidinediones: a meta-analysis of randomised clinical trials. Lancet 2007;370:1129-36.

10. Tzoulaki I, Molokhia M, Curcin V, et al. Risk of cardiovascular disease and all cause mortality among patients with type 2 diabetes prescribed oral antidiabetes drugs: retrospective cohort study using UK general practice research database. BMJ 2009;339:b4731.

11. Home PD, Pocock SJ, Beck-Nielsen H, et al. Rosiglitazone evaluated for cardiovascular outcomes in oral agent combination therapy for type 2 diabetes (RECORD): a multicentre, randomised, open-label trial. Lancet 2009;373:2125-35.

12. Diamond GA, Bax L, Kaul S. Uncertain effects of rosiglitazone on the risk for myocardial infarction and cardiovascular death. Ann Intern Med 2007;147:578-81.

13. Nissen SE, Wolski K. Effect of rosiglitazone on the risk of myocardial infarction and death from cardiovascular causes. $N$ Engl J Med 2007;356:2457-71.
14. Maru S, Koch GG, Stender M, et al. Antidiabetic drugs and heart failure risk in patients with type 2 diabetes in the U.K. primary care setting. Diabetes Care 2005;28:20-6.

15. Bell DS. Do sulfonylurea drugs increase the risk of cardiac events? CMAJ 2006;174:185-6.

16. Scirica BM, Bhatt DL, Braunwald E, et al. Saxagliptin and cardiovascular outcomes in patients with type 2 diabetes mellitus. N Engl J Med 2013;369:1317-26.

17. Fu AZ, Johnston SS, Ghannam A, et al. Association between hospitalization for heart failure and dipeptidyl peptidase 4 inhibitors in patients with type 2 diabetes: an observational study. Diabetes Care 2016;39:726-34.

18. Food and Drug Administration. Guidance for industry: diabetes mellitus - evaluating cardiovascular risk in new antidiabetic therapies to treat type 2 diabetes Maryland, 2008. https://www.fda. gov/downloads/Drugs/Guidances/ucm071627.pdf

19. Palmer SC, Mavridis D, Nicolucci A, et al. Comparison of clinical outcomes and adverse events associated with glucose-lowering drugs in patients with type 2 diabetes: a meta-analysis. JAMA 2016;316:313-24.

20. Faraoni D, Schaefer ST. Randomized controlled trials vs observational studies: why not just live together? BMC Anesthesiol 2016;16:102.

21. Rouse B, Chaimani A, Li T. Network meta-analysis: an introduction for clinicians. Intern Emerg Med 2017;12:103-11.

22. Liberati $A$, et al. The PRISMA statement for reporting systematic reviews and meta-analyses of studies that evaluate health care interventions: explanation and elaboration. Ann Intern Med 2009;151:W-94.

23. Shamseer L, Moher D, Clarke M, et al. Preferred reporting items for systematic review and meta-analysis protocols (PRISMA-P) 2015: elaboration and explanation. BMJ 2015;349:g7647.

24. Wannamethee SG, Shaper AG, Lennon L. Cardiovascular disease incidence and mortality in older men with diabetes and in men with coronary heart disease. Heart 2004;90:1398-403.

25. Higgins JP, Altman DG, Gøtzsche PC, et al. The Cochrane Collaboration's tool for assessing risk of bias in randomised trials. BMJ 2011;343:d5928.

26. von Elm E, Altman DG, Egger M, et al. Strengthening the reporting of observational studies in epidemiology (STROBE) statement: guidelines for reporting observational studies. BMJ 2007;335:806-8.

27. White IR, Barrett JK, Jackson D, et al. Consistency and inconsistency in network meta-analysis: model estimation using multivariate metaregression. Res Synth Methods 2012;3:111-25.

28. White IR. Network meta-analysis. Stata J 2015;15:951-85

29. Chaimani A, Salanti G. Visualizing assumptions and results in network meta-analysis: the network graphs package. Stata $J$ 2015;15:905-50.

30. Cochran WG. The combination of estimates from different experiments. Biometrics 1954;10:101-29.

31. Higgins JP, Thompson SG, Deeks JJ, et al. Measuring inconsistency in meta-analyses. BMJ 2003;327:557-60.

32. Higgins JP, Thompson SG, Spiegelhalter DJ. A re-evaluation of random-effects meta-analysis. J $R$ Stat Soc Ser A Stat Soc 2009;172:137-59.

33. Jansen JP, Naci $\mathrm{H}$. Is network meta-analysis as valid as standard pairwise meta-analysis? It all depends on the distribution of effect modifiers. BMC Med 2013;11:159.

34. Chaimani A, Higgins JP, Mavridis D, et al. Graphical tools for network meta-analysis in STATA. PLoS One 2013;8:e76654.

35. Salanti G, Ades AE, loannidis JP. Graphical methods and numerical summaries for presenting results from multiple-treatment metaanalysis: an overview and tutorial. J Clin Epidemiol 2011;64:163-71.

36. Salanti G, Del Giovane C, Chaimani A, et al. Evaluating the quality of evidence from a network meta-analysis. PLoS One 2014;9:e99682. 